\title{
Level set detected masses in digital mammograms
}

\author{
R. B. Dubey ${ }^{1}$, M. Hanmandlu ${ }^{2}$ and S. K. Gupta ${ }^{3}$ \\ ${ }^{1}$ Dept. of E \& IE, Apeejay College of Engg., Sohna, Gurgaon; ${ }^{2}$ Dept. of EE, IIT, Delhi, India. \\ .$^{3}$ Vaish College of Engg, Rohtak, Haryana, India \\ rbdubeyster@gmail.com
}

\begin{abstract}
Breast cancer is the leading cause of death among woman. Currently X-ray mammography is the most widely used method for early detection of breast cancer. Many computer aided techniques are available to assist the radiologist in taking crucial decisions. A method uses level set for segmentation of masses in digital mammograms is introduced. This method uses the Gaussian filter for smoothing the image and noise reduction. Level set methods offer a powerful approach for the medical image segmentation since it can handle any of the cavities, concavities, convolution, splitting or merging. However, this method requires specifying initial curves and can only provide good results if these curves are placed near symmetrically with respect to the object boundary. The results of experimental study indicate that our scheme can provide useful contour extraction for mass structure.
\end{abstract}

Keywords. Breast cancer, level set, mammograms, Gaussian filter.

\section{Introduction}

Breast cancer is the second most common cause of cancer death in women (after lung cancer) and the main cause of death in women ages 45 to 55 . Every year, 205,000 American women are diagnosed with breast cancer and $>40,000$ die from this disease. X-ray mammography is the current, clinical Gold Standard for the detection of breast cancer. Though it is inexpensive and works fairly well in postmenopausal women, it is less sensitive in premenopausal women. In addition, X-ray exposure can cause cancer (Boyle et al., 2005).

Segmentation is an important technique used in image processing to identify the objects. As we work with data from simulations, observations, and experiments, it of interest to apply segmentation techniques in a robust and efficient way to image and mesh data. Mesh data is frequently unstructured; this precludes the direct application of techniques that were originally developed for more structured image data. One solution to this problem is the use of techniques such as level sets or implicit active contours. The idea behind active contours or deformable models for image segmentation is quite simple. The user specifies an initial guess for the contour, which is then moved by image driven forces to the boundaries of the desired objects. In such models, two types of forces are considered-the internal forces, defined within the curve, are designed to keep the model smooth during the deformation process, while the external forces, which are computed from the underlying image data, are defined to move the model toward an object boundary or other desired features within the image (Kupinski et al., 1998; Hanmandlu et al., 2008).

There are two forms of deformable models. In the parametric form, also referred to as snakes, an explicit parametric representation of the curve is used. This form is not only compact, but is robust to both image noise and boundary gaps as it constrains the extracted boundaries to be smooth. However, it can severely restrict the degree of topological adaptability of the model, especially if the deformation involves splitting or merging of parts. In contrast, the implicit deformable models, also called implicit active contours or level sets, are designed to handle topological changes naturally. However, unlike the parametric form, they are not robust to boundary gaps and suffer from several other deficiencies as well (Suri et al., 2001; 2002). Among the various image segmentation techniques, level set methods offer a powerful approach for the image segmentation since it can handle any of the cavities, concavities, splitting/merging, and convolution. It has been used in wide fields including the medical image processing. However, despite of all the advantages, which this method can provide, it requires the prior choice of the most important parameters such as the initial location of seed point, the appropriate propagation speed function and the degree of smoothness. The traditional methods only depend on the contrast of the points located near the object boundaries, which cannot be used for the accurately results of complex medical image segmentation (Millandi et al., 1995; Sethian, 1996).
Research article

CIndian Society for Education and Environment (iSee)
"Digital mammograms" http://www.indjst.org
Dubey et al. Indian J.Sci.Technol. 
The paper encompasses proposed methodology and its implementation; the results of experimental work and conclusions and future plan of work.

\section{The proposed methodology and its implementation}

Our main motivation for investigating level set techniques was to better understand their pros and cons relative to the more traditional image segmentation techniques. Some of early work is summarized in (Weeratunga et al., 2004). Figure1 shows the results obtained using level sets to segment a grain of pollen from the background. To obtain the outer boundary of the grain, we started with an initial level set at the boundary of the image. To obtain the structures on the inside of the pollen grain, we started with an initial level set that was a closed curve around a point on the inside. This curve evolved to identify the boundaries of all the spines inside the grain. The contours generated by the level sets are closed contours. This is in contrast with edge detection methods such as the canny technique that typically requires a post-processing step to generate closed contours. However, relative to the canny method, the level sets are far more computationally intensive, especially when used to segment a complex image such as the inside of the pollen grain (Dzung et al., 2003; Suri et al., 2001; 2002).

Fig. 1: Panels (a) and (b) illustrate the use of level sets in segmenting a pollen grain and panel (c) is the output of the canny edge detector, with the edges in black.

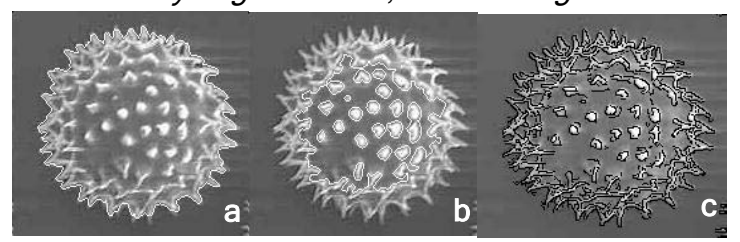

Let us suppose an interface separating one region from another and a speed that tells us how to move each

Fig. 2: An interface separating one region from another.
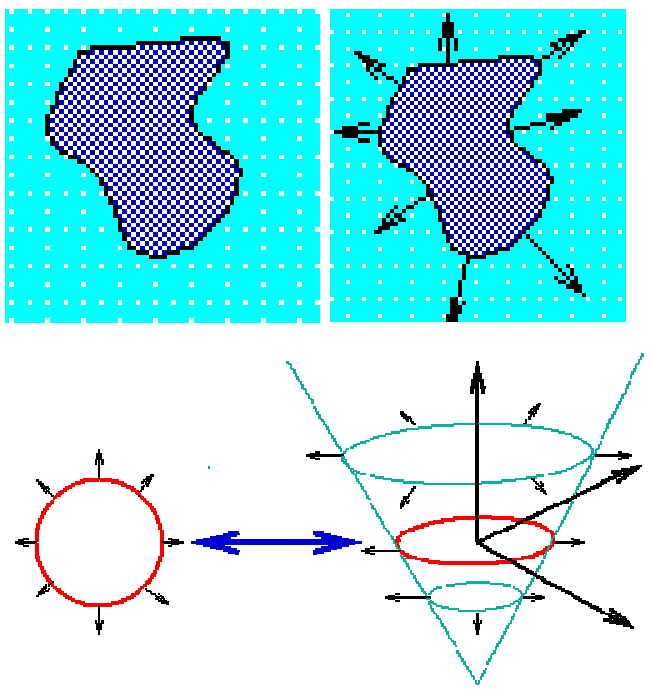

point of the interface. In the figure 2 below, a black curve separates a dark blue inside from a light blue outside, and at each point of the black curve the speed is given. This speed can depend on a variety of physical effects. For example imagine that the dark blue is ice and the light blue is water. Then the boundary can shrink as the ice melts, or grow as the ice freezes; the speed then depends on the temperature jump between the two.

Fig. 3: Level set model represent curves and surfaces implicitly using grey scale images.
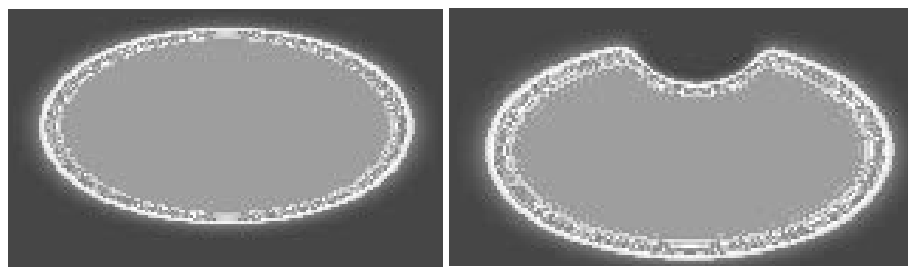

Most numerical techniques rely on markers, which try to track the motion of the boundary by breaking it up into buoys that are connected by pieces of rope. The idea is to move each buoy under the speed and rely on the connecting ropes to keep things straight. The hope is that more buoys will make the answer more accurate. Unfortunately, things get pretty dicey if the buoys try to cross over themselves, or if the shape tries to break into two; in these cases, it is very hard to keep the connecting ropes organized. In three dimensions, following a surface like a breaking ocean wave is particular tough (Cardoso et al., 2005; Chitre, 1994; Weeratunga et al., 2004; Bischoff et al., 2002; 2003).

Rather than follow the interface itself, the level set approach introduced by Osher and Sethian instead takes the original curve to build a surface. That cone-shaped surface, which is shown in blue-green on the right below, has a great property; it intersects the xy plane exactly where the curve sits. The blue-green surface on the right below is called the level set function, because it accepts as input any point in the plane and hands back its height as output. The red front is called the zero level set, because it is the collection of all points that are at height zero. Level set methods are designed for problems in which the speed function can be positive in some places are negative in others, so that the front can move forwards in some places and backwards in others. Our aim is the development of robust and edible segmentation Method on images with non sharp segment boundaries as shown in fig. 3 (Sethian, 1982).

In contrast to the approaches that basically operate at the granularity of individual voxels without any notion of the surface of the segmented object, maintaining and modifying, i.e., deforming, actual object surfaces has many advantages. A comprehensive survey of deformable models in medical image analysis has been presented by Mclnerney and Terzopoulos (1996), although they do not consider implicit representations of the model, i.e., level set methods. A very good
Research article

CIndian Society for Education and Environment (iSee)
"Digital mammograms" http://www.indjst.org
Dubey et al. Indian J.Sci.Technol. 
introduction to level set methods, including their application to image segmentation, can be found in the book by Osher and Fedkiw (2003). Leventon (2000) employs statistical models to direct the evolution of a surface with local and global priors towards the final segmentation, a method that represents a hybrid of using a priori knowledge obtained from a training set and level set methods.

Where active contour models maintain an explicit representation of object contours, curves and surfaces can naturally also be represented in implicit form, turning them into deformable is surfaces. Level set methods represent an implicit surface via a grid of scalar function samples, which most commonly are the signed distances to the surface. Level set methods (Whitker et al., 2001; Sethian, 1982; Osher et al., 2003) have established themselves as a very powerful framework for maintaining and updating interfaces, i.e., hyper-surfaces, by solving partial differential equations (PDEs). Surface motion is the result of updating the distance values to the surface according to a speed function that moves the surface into the direction of the implicit surface normal, i.e., the gradient of the scalar field.

A Common speed functions includes the local curvature of the model (Malladi \& Sethian, 1996). In segmentation with level set methods, a central part of the speed function is a measure for intensity differences between adjacent voxels in the original volume (Osher et al., 2003).For rendering, the actual surface must be extracted or rendered directly from the sampled implicit representation, e.g., generating a mesh via marching cubes or one of its variants, or rendering a view of the surface without generating any geometry via ray-casting. A very important difference of level set methods and other deformable surface models, including snakes, is that surfaces represented implicitly can easily change their topology, e.g., break apart or merge, and develop sharp features. In each step, only the scalar sample values are updated, possibly changing the surfaces topology implicitly. A major drawback of level set methods is that the quality of the resulting segmentation often depends significantly on parameter tuning (Lefohn et al., 2003), a problem which can be tackled by computing and visualizing the level set solution interactively. Preusser and Rumpf (2002) present a multiscale method for nonlinear diffusion of 2D geometry. Weeratunga and Kamath (2004) evaluate the use of implicit active contours for image segmentation and compare them with traditional edge detectors. Whitaker et al. (2001) present the application of level set methods to the segmentation of biological data sets. Leventon (2000) combine intensity and curvature information obtained from a training set with level set evolution in order to reach the final segmentation. Level set topology can be controlled with sparse information added to the volume in order to make use of surface genus information known a priori (Goldenberg et al., 2002) detect the inner (center) and the outer (right) boundary of the gray matter of the brain (left), and initialize two coupled level sets from these boundaries. The coupled surfaces are then evolved simultaneously (Bischoff et al., 2002; 2004).

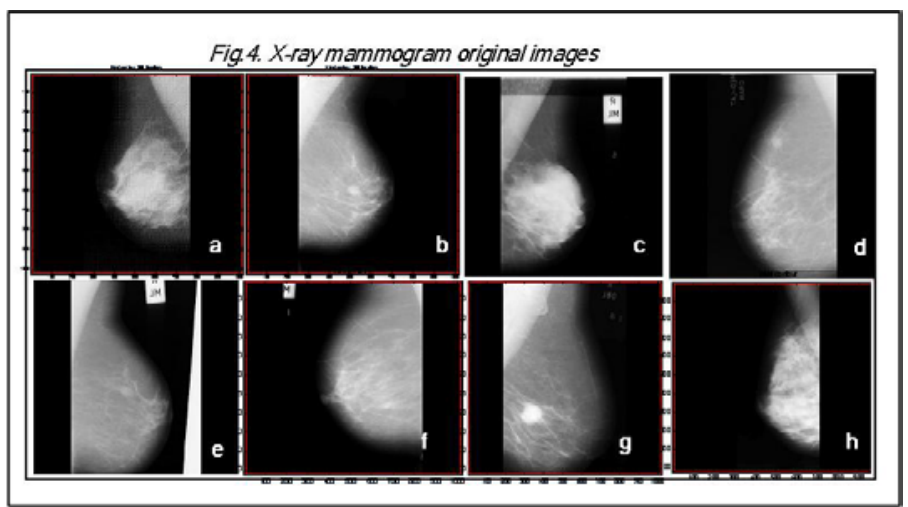

\section{Algorithm}

The level set method has been used to capture rather than track interfaces. Because the method is stable, the equations are not unnecessarily stiff, geometric quantities such as curvature become easy to compute, and three dimensional problems present no difficulties, this technique has been used in a wide collection of problems involving moving interfaces, including the generation of minimal surfaces, singularities and geodesics in moving curves and surfaces, flame propagation, etching, deposition and lithography calculations, crystal growth, and grid generation (Hua et al., 2003 ).

They embed the initial position of the moving interface $C_{0}(x)$ as the zero level set of a higher dimensional function $\phi$, the signed distance to $C_{0}$, and link the evolution of this new function $\phi$ to the evolution of the interface itself through a time-dependent initial value problem. At each time, the contour $C(\mathrm{t})$ is given by the zero level set of $\phi$. This condition states that

$$
(C(t), t)=0 \Rightarrow t+\nabla(C(t), t) \cdot \partial C / \partial t=0
$$

Since $\partial C / \partial t=F_{n}$ and outward normal vector is given by $\nabla \phi /|\nabla \phi|$, this yields the following evolution equation for $\phi$ :

$$
\begin{array}{r}
t+F|\nabla|=0 \\
(x, 0)=\mathrm{C}_{0}(\mathrm{x})
\end{array}
$$

For reasons of causality, it is possible to restrain the computation domain to a band of cells around the zerolevel set of $\phi(\mathrm{x}, t)$, for the decrease of the computational cost. Classical approaches are referred to as narrowband methods.

According to the above discussion, the level set method requires specifying initial curves and can only provide good results if these curves are placed near symmetrically with respect to the object boundary. When using the level set method in image segmentation, an initial front should be chose appropriately, and let it 
propagate with a speed function that stops the motion when the boundary is reached. So the initial front and the speed function are important ones to decide the accuracy of the final segmentation. Thus, level set segmentation is not sufficient for the segmentation of complex medical images; they must be combined with powerful initialization techniques in order to produce successful segmentation (Hua et al., 2003).

\section{Results}

The images considered here are taken from MIAS database. We introduce a novel segmentation approach for mammogram images and investigate its application to the detection of region of interest (ROI) which includes both masses and the pectoral muscles. In mammograms masses are assumed to be distinctive regions that are relatively brighter than the surrounding background, while the pectoral muscles appears to be more uniformly bright and to appear in a predictable location. In Fig.4 (a, b, c, d, e, f, g, h) and in Fig. 5 (a, b, c, d, e, f, g, h), x-ray mammograms original images and images after Gaussian filter smoothing are shown. Fig. 6 shows the segmented images after the application of level set and $\mathrm{ROI}$. The results are validated by visual inspection by an expert radiologist.
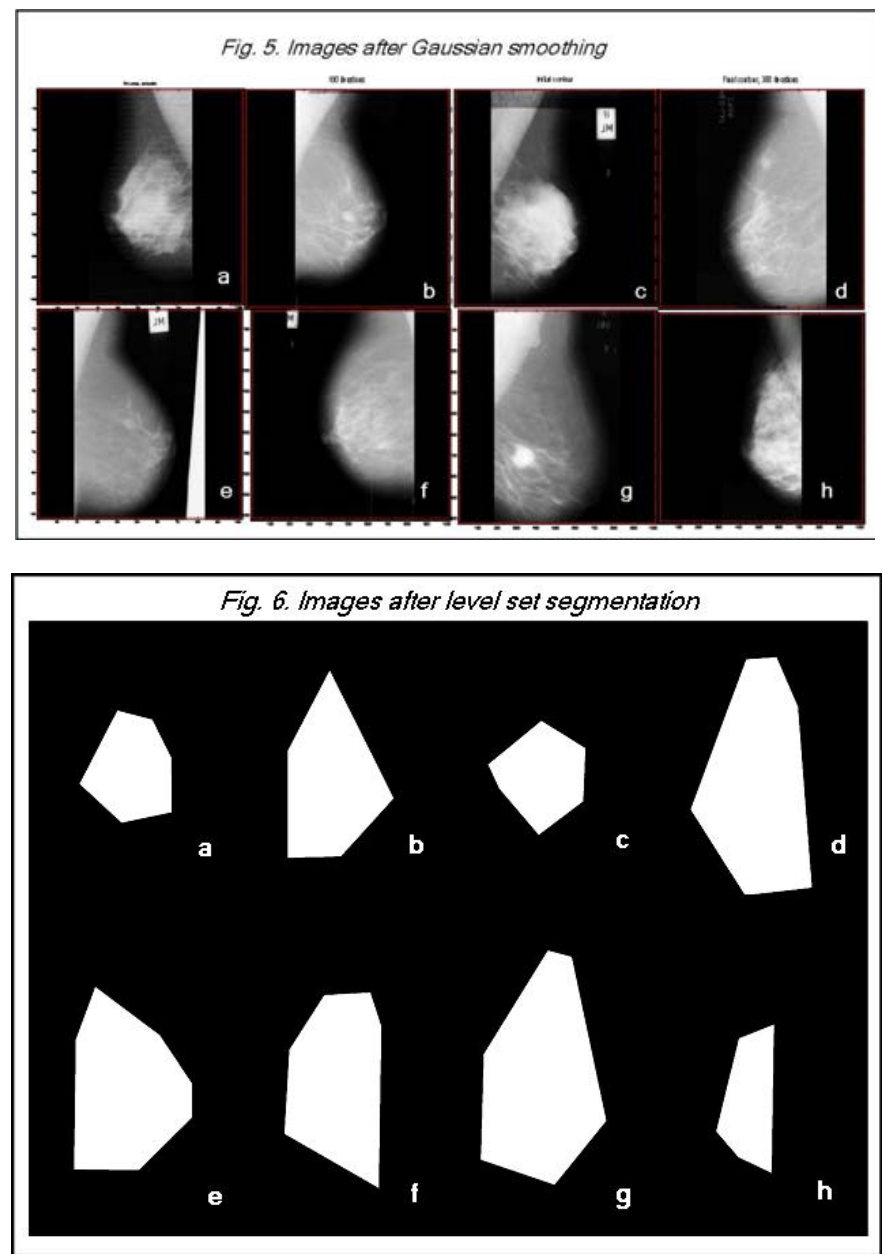

\section{Conclusions and future work}

The used algorithm is suited for gray scale images. As mammograms images are simple but more affected by noise and hence preprocessing of image is very essential. In our experiments the used algorithms works well on mammograms images. The results show the exact boundary of abnormal growth or lesions and hence this technique is comparatively superior to other methods. We believe that our application allows the radiologist to perform a more in depth exploration of the breast and considerably reduces the usual workflow time. We think this will lead to a more accurate diagnosis.

In the domain of medical image analysis, timeconsuming algorithms are synonymous with noninteractive methods and are therefore limited to a very small number of specific applications. In other words, the computation times of level sets are an obstacle to a wider use of these methods. Our future work includes the mammogram image segmentation by combine use of watershed and level set methods. Further work on this hybrid approach is on progress. We are planning to present a fast hybrid segmentation algorithm that integrates watershed transformation and level set method. Here user may select the iteration at which the resulting segmentation is acceptable. It is predicted that the algorithm can provide satisfactory results both with respect to segmentation performance and execution times for 2-D and 3-D cases. Also we are planning to develop a CAD system for early detection of breast cancer.

\section{References}

1. American cancer society (2004) Breast cancer facts and figure, ACS Web site.

2. Bischoff S and Kobbelt L (2002) Isosurface reconstruction with topology control. Proceedings of Pacific Graphics, pp. 246-255.

3. Bischoff $S$ and Kobbelt L (2003) Sub-voxel topology control for level set surfaces. Proceedings of Eurographics, pp. 273-280.

4. Bischoff $S$ and Kobbelt L (2004) topologically correct extraction of the cortical surface of a brain using levelset methods. Proceedings of BVM.

5. Boyle P and Ferly J (2005) Cancer incidence and morality in Europe. Ann. Oncology. 16(3), 481-488.

6. Cardoso JS, Costa JFPD and Cardoso MJ (2005) SVMs applied to objective Aesthetic evaluation of conservative breast cancer treatment. IEEE Proceedings of Int. joint conf. on NN, pp.2481-2486.

7. Chitre Y, Dhawan AP and Moskowtz M (1994) ANN based classification of mammography microclssification using image structure and cluster features. IEEE.

8. Goldenberg R, Kimmel R, Rivlin E and Rudzsky M (2002) Cortex segmentation: A fast variational geometric approach. IEEE Transactions on Medical Imaging. 21(2), 1544-1551.
Research article

CIndian Society for Education and Environment (iSee)
"Digital mammograms" http://www.indjst.org
Dubey et al. Indian J.Sci.Technol. 
9. Hanmandlu M, Vineel D and Singh G (2008) Computer aided fuzzy based technique for detection of suspicious masses and microclassifications from mammographic images. //CA/-07, pp.344-358.

10. Hua LI, LElmoataz A, Fadilia J and Su R (2003) An improved image segmentation approach based on level set and mathematical morphology. Proceedings of SPIE, 5286, 851.

11. Kupinski MA and Giger M (1998) Automated seeded lesion segmentation on digital mammograms. IEEE Trans. Med. Imaging. 17(4), 510-517.

12. Lefohn A, Cates J and Whitaker R (2003) Interactive, gpu-based level sets for $3 d$ brain tumor segmentation. Proceedings of Medical Image computing and Computer Assisted Intervention (MICCAI).

13. Leventon M (2000) Statistical Models for Medical Image Analysis. Ph.D. thesis, MIT.

14. Malladi R and Sethian J (1996) Image processing: Flows under $\mathrm{min} / \mathrm{max}$ curvature and mean curvature.Graphical Models and Image Processing, 58 (2) pp. 127-141.

15. Malladi R, Sethian J and Vemuri BC (1995) Shape Modeling with Front Propagation: A Level Set Approach. IEEE Trans. PAMI, vol. 17, pp. 158-175.

16. Mclnerney $T$ and Terzopoulos D (1996) Deformable models in medical image analysis: A survey, Medical Image Analysis. 1(2), 91-108.

17. Osher S and Fedkiw R (2003) Level Set Methods and Dynamic Implicit Surfaces. Springer Verlag, New York.

18. Pham DL, Xu C and Prince JL (2000) Current methods in medical image segmentation. Ann. Rev. Biomed. Eng., vol. 2, 315-337.

19. Preusser T and Rumpf M (2002) A level set method for anisotropic geometric diffusion in $3 d$ image processing. SIAM J. Applied Mathematics, 62 (5) pp. 1772-1793.

20. Rafael C, Gonzalez and Richard Woods E (2003) Digital Image Processing, $2^{\text {nd }}$ edition.

21. Sethian J (1982) An analysis of flame propagation, Ph.D. Thesis, University of California at Berkeley.

22. Sethian JA (1996) Level Set Methods: Evolving Interfaces in Geometry, Fluid Mechanics. Computer Vision and Material Science, Cambridge University, UK.

23. Sonka M, Hlavac V and Boyle R (2007) Image Processing: Analysis and Machine Vision, $3^{\text {rd }}$ edition.

24. Suri JS, Liu KC, Singh S, Laxminarayana SN and Reden L (2002) Shape recovery algorithms using level sets for 2D and 3D medical imagery: A state-ofthe-art review. IEEE Trans. Info. Tech. in Biomed. 6(1), 8-28.

25. Suri et al., (2001) Fast MR Brain Segmentation using Regional Level Sets. Intern. Jou. of Engg. Med. and Biol. 20(4), 84-95.

26. Weeratunga $S$ and Kamath $C$ (2004) An investigation of implicit active contours for scientific image
Vol. 3 No. 1 (Jan 2010)

ISSN: 0974- 6846

segmentation, Video Communications and Image Processing. SPIE Elec. Imaging. 5308, 210-221.

27. Weeratunga $S$ and Kamath $C$ (2004) An investigation of implicit active contours for scientific image segmentation. Proc. of Visual Commun. \& Image Processing Conf.

28. Whitaker R, Breen D, Museth K and Soni N (2001) A framework for level set segmentation of volume datasets. Proc. Intl. Workshop on Volume Graphics. pp: 159-168.

29. Whitaker R, Breen D, Museth K and Soni N (2001) segmentation of biological volume datasets using a level set framework. Proc. Intl. Workshop on Volume Graphics. pp: 249-263.
Research article

CIndian Society for Education and Environment (iSee)
"Digital mammograms" http://www.indjst.org
Dubey et al. Indian J.Sci.Technol. 